\title{
Tarrius Alain, Étrangers de passage. Poor to poor, peer
} to peer et La mondialisation criminelle

Fatima Qacha

\section{(2) OpenEdition}

Édition électronique

URL : https://journals.openedition.org/remi/7558

DOI : $10.4000 /$ remi.7558

ISSN : $1777-5418$

Éditeur

Université de Poitiers

\section{Édition imprimée}

Date de publication : 1 mars 2016

Pagination : 229-232

ISBN : 979-10-90426-27-6

ISSN : 0765-0752

Référence électronique

Fatima Qacha, «Tarrius Alain, Étrangers de passage. Poor to poor, peer to peer et La mondialisation criminelle », Revue européenne des migrations internationales [En ligne], vol. $32-n^{\circ} 1$ | 2016, mis en ligne le 01 mars 2016, consulté le 14 avril 2022. URL : http://journals.openedition.org/remi/7558 ; DOI : https://doi.org/10.4000/remi.7558 


\section{Notes de lecture}

Tarrius, Alain

Étrangers de passage. Poor to poor, peer to peer. - La Tour d'Aigues : L'Aube, 2015. $175 \mathrm{p}$.

ISBN : 978-2815-911-44-3

La mondialisation criminelle. - La Tour

d'Aigues : L'Aube, 2015. - $150 \mathrm{p}$.

ISBN : 978-2815-911-47-4

L'auteur avait prévu la publication d'un seul ouvrage de 330 pages qu'il intitulait Deux mondialisations souterraines en Europe, sous-titré, Livre I : le fétichisme de la marchandise entre pauvres et Livre II : transmigrations des femmes pour le travail du sexe et la vente des drogues. L'éditeur, Jean Viard, directeur des éditions de l'Aube, lui proposa d'éditer séparément les deux livres, car si les deux formes de mondialisation étudiées laissent apparaître quelques liens, en particulier par des blanchiments croisés d'argent, les logiques d'acteurs sont différentes. Les " transmigrants marchands-entre-pauvres " manifestent des initiatives de nature autre que celles des " transmigrantes marchandisées " pour le travail du sexe, même si de nombreux échanges les mettent en présence le long des territoires circulatoires de leurs tournées transeuropéennes. II reste toutefois utile de disposer des deux volumes : les renvois de l'un vers l'autre sont fréquents, le chercheur ayant pratiqué des terrains communs aux deux types de circulation.

Dans Étrangers de passage, Alain Tarrius expose une synthèse de ses travaux sur ceux qu'il a dénommés, dès 1987 et jusqu'en 1999, "les migrants nomades ", puis à partir de 2001 et après avoir décrit les formes d'une mondialisation entre pauvres - ou poor to poor - ou encore mondialisation par le bas, les " transmigrants ". II témoigne ainsi de l'apparition dans des nations européennes, sur le mode de centralités souterraines, de vastes emplacements marchands dans les années 1980 à partir d'initiatives de collectifs de migrants originaires de nations euro-périphériques, puis, à partir des années 1990, de la substitution des places marchandes par des multi-centralités de réseaux. Les initiatives de populations turques et marocaines structurent alors les territoires circulatoires transeuropéens nord et sud, densifiés dans les années 2000 par les circulations moyen-orientales amalgamant le long de leurs parcours des nationaux balkaniques.

Dans cet ouvrage, le chercheur présente ses travaux de 2008 à 2015 sur les territoires circulatoires nord méditerranéens, "route des Sultans " de la mer Noire à la mer Adriatique, « route en pointillés " de quartiers de relégation en ghettos urbains dans les villes italiennes, françaises et espagnoles. II prend à témoin les populations transmigrantes baloutches, iraniennes et afghanes, du commerce des produits électroniques d'entrée de gamme " free tax, passed by Dubaï " des grandes firmes du Sud-Est asiatique, en tournées de six mois de chez eux à chez eux par la voie méditerranéenne. Nombreux, plusieurs centaines de milliers, et invisibles parmi des millions de voyageurs, car en tournées sous visa touristique, ces étrangers de passage qui échappent au cadrage statistique spécifique n'en transforment pas moins les contours de villes telles queTrabzon, port turc emblématique de la mer Noire, Sofia, Tarente et Alicante. Sa redéfinition, à l'échelle de réseaux internationaux, de la notion de Park de moral area - ou " espace de mœurs " - lui est alors utile pour décrire le rôle des transmigrants dans les agencements de ces villes et l'apparition de métissages ou de cosmopolitismes nouveaux permis par 
les échanges marchands indifférenciés : "les pauvres, dès lors que l'opportunité leur est donnée de se reconnaître comme collectif d'usagers dans la ville, entrent sans réticence dans la complexité des rapports d'altérité et exposent leur cosmopolitisme, de circulation et de voisinage urbain ". Depuis les années 1980, Alain Tarrius propose deux autres notions clefs pour aborder ses terrains ; d'abord le " paradigme de la mobilité ", notion d'usage méthodologique qui lui permet d'identifier les rapports sociaux originaux, interindividuels et collectifs, institués par les migrants de passage comme autant d'interactions entre eux et avec les habitants sédentaires. Ensuite les " territoires circulatoires ", topique souple, évolutive, qui concrétise les continuités sociales et territoriales créées par ces populations à travers les nations. Ces trois notions sont redéfinies par les nouveaux apports de terrain au fur et à mesure de l'enchaînement des recherches de l'auteur. Deux annexes au premier ouvrage en détaillent précisément le contenu et les usages.

Deux préalables issus des travaux antérieurs du chercheur sont appelés pour les cadrages contextuels. D'une part, les importants enjeux financiers attachés à ces commerces souterrains comme résultats des connivences entre acteurs majeurs des économies criminelles de la drogue et des économies officielles, les grandes firmes du Sud-Est asiatique : la seule filière souterraine des produits électroniques à partir des importations émiraties passant par la mer Noire et la voie méditerranéenne écoule environ 6 milliards de dollars avancés aux transmigrants moyen-orientaux par les milieux criminels russo-italiens des trafics de l'héroïne et des femmes. Les stratégies de la mondialisation entre pauvres, ou poor to poor, déployées par les grands opérateurs de l'électronique du Sud-Est asiatique, facilitatrices des commerces des transmigrants qui se chargent de marchandises directement livrées des Émirats non seulement dans les ports de la mer Noire, mais encore lors de leurs étapes de
Durrës, sur l'Adriatique, et de Valencia, ne concernent que des populations clientes de pauvres et ne concurrencent pas les distributions officielles. Nous sommes bien loin des marchés et bazars de vêtements et colifichets asiatiques. D'autre part, second préalable, le chercheur refuse de se cantonner aux populations de nations pauvres extérieures à l'espace Schengen : l'économie entre pauvres n'est pas un attribut exclusif de nations sous-développées. Au contraire, nous suivons de ville à ville, de nation à nation, de continent à continent, les parcours de ces hordes de saute-frontières. Mais surtout, nous voyons, à partir des villes européennes, comment deux séries de phénomènes sociétalement structurants se déploient : en premier lieu la suggestion, par l'unité transfrontalière qu'opère le territoire circulatoire européen, de l'existence de peuples sans nation, en particulier turc et marocain, agrégeant et dynamisant les immigrations sédentaires dispersées à travers I'Europe depuis plusieurs décennies. II n'est pas rare qu'une branche familiale de Marocains installée en Espagne entretienne des relations étroites avec d'autres branches en France, en Italie, en Allemagne et en Belgique. Des mobilités incessantes opèrent comme recours en cas de chômage ou encore pour la santé, etc. Les frontières ne sont plus celles des nations, mais de l'entité sociale marocaine. En second lieu, la mobilité internationale des transmigrants opère comme " ressource de sortie par le bas " pour des jeunes des enclaves urbaines des villes européennes. Le constat du passage du classique paradigme immigration/intégration à celui transmigration/mondialisation correspond, au fond, à un changement de focale marxiste. "Au revoir le Marx du moteur unique de la migration comme mobilisation internationale de la force de travail. Bonjour la remarquable intuition de Marx sur le fétichisme de la marchandise en situation de globalisation libérale " (p. 5). La mobilisation internationale et les assignations sédentaires des immigrants 
qu'elle a exigées ont produit les ghettos urbains. Le fétichisme de la marchandise promeut les migrants en entrepreneurs mobiles au service des économies marchandes ultralibérales : actualisation de l'exploitation des migrants pauvres, certainement, mais vécue comme libération d'initiatives par ceux-ci. C'est ainsi que la plupart des transmigrants déclarent ne jamais quitter leur " chez soi ", qu'ils enrichissent tournée après tournée. Les multiples étapes sur les vastes territoires des circulations deviennent socialement proches, organisées en voisinages qui redéfinissent les notions d'origine et de destination.

Les difficultés de lecture de ce livre tiennent à la souplesse, la déclinaison constante des approches compréhensives développées par Alain Tarrius. La redéfinition des notions clefs, lors de ses incessants allers-retours entre terrains et univers notionnel, déroute le lecteur qui n'est pas au fait de cette gymnastique bien en phase avec les recommandations de Weber, Simmel, Park, Goffman, Barel, Lourau, Hanertz qu'il cite fréquemment, parmi d'autres chercheurs plus contemporains, Ma Mung, Agier, Diminescu, Kokoreff, Boubeker, etc. Le " mimétisme " avec les populations transmigrantes et donc " l'enquête en mobilité " engendrent d'importantes contraintes, que comprennent peu les laboratoires gestionnaires, mais qui permettent à Alain Tarrius de décrire les rapports sociaux originaux produits dans les temporalités singulières des territoires circulatoires. Le choix de « situations révélatrices " (Goffman) livre au lecteur des scènes heureusement choisies et bien enracinées dans la réalité des interactions, occasions de renouvellement de l'intérêt de la lecture.

Dans La mondialisation criminelle, il ne s'agit plus de synthèses ni d'actualisations de recherches menées depuis trois décennies, mais d'une enquête, d'un enchaînement de terrains, de situations, qui éclairent crûment le phénomène étudié : le trafic de femmes, pour le travail du sexe, et de drogues depuis les mêmes entours, de la mer Noire aux nations de I'espace Schengen.

Le fétichisme de la marchandise femmes-drogues est porté à son plus haut niveau par la libéralisation-globalisation des milieux criminels. L'auteur étudie ici les filières transeuropéennes russoitaliennes et leur grande proximité avec les territoires circulatoires des transmigrants du poor to poor. Les origines des " marchandises " (Balkans et Caucase pour les femmes, Afghanistan, Turquie, Géorgie et Russie caucasienne pour les drogues opiacées), des recruteurs (Albanais, Géorgiens, Serbes), des espaces de distribution ou moral areas (Italie du Sud, Levant espagnol, routes françaises, nations permissives nord-européennes) se diversifient à l'échelle de la globalisation du marché. Transmigrantes, ces femmes le sont assurément, qui parcourent en cinq ou six années l'ensemble des moral areas. Évidemment les temporalités ne sont pas les mêmes que celles des transmigrants du poor to poor, mais les cosmopolitismes de route sont proches et les investissements au lieu d'origine souvent identiques. Alain Tarrius suit particulièrement une cohorte de femmes accompagnées de parentèles, frères, sœurs, jeunes parents et proches amis du village ou du quartier d'origine. Ces accompagnateurs travaillent et habitent non loin des clubs prostitutionnels ; ils maintiennent un lien constant avec le milieu d'origine et sont les acteurs résolus de la réalisation du projet de retour. Ses enquêtes, à partir de l'aide de commissaires territoriaux espagnols, qui exercent la tolérance sur les clubs prostitutionnels, lui permettent d'évaluer à 10800 le nombre des femmes des Balkans et du Caucase qui travaillent dans le Levant ibérique, puis d'évaluer les importants blanchiments des revenus des drogues par le travail légal du sexe. Une enquête auprès de 120 femmes, entre 2009 et 2011, lui permet de maintenir un réseau de quarante-sept informatrices, via Skype, et 
d'enquêter auprès de dix-sept d'entre elles le long des routes et autoroutes françaises. II y gagnera dans les aires de service le surnom de " mac boiteux " (en raison de sa claudication) fortement contrôlé lors de ses terrains, non par des personnages des milieux qui exploitent ces femmes, mais par divers responsables de commerces et d'exécutifs politiques locaux. C'est ainsi que lors d'une passionnante enquête sur les passages drogues et femmes de la frontière espagnole du Perthus, il met précisément en évidence le processus d'expansion spatiale tributaire de la moral area de La Junquera, au Perthus : I'omniprésent clientélisme politique des exécutifs départemental et municipal de Perpignan, ses contentions, omertas et violences, fait le lit des milieux mafieux russo-italiens. Une " communauté morale " s'établit entre les deux espaces frontaliers qu'unissent les multiples mobilités des hommes, consommateurs et trafiquants, et de l'argent des bourgeois rentiers, malgré l'apparence contradictoire des législations sur le travail du sexe. Des adolescent-e-s des collèges, lycées, centres de formation de ce département deviennent des proies faciles pour la pénétration des drogues chimiques, et certain-e-s d'entre eux-elles sont happé-e-s par le dispositif prostitutionnel espagnol. Le chercheur déploie méticuleusement, pour cette enquête sur la constitution et le fonctionnement d'une moral area transfrontalière, des méthodologies proches de celles proposées par l'analyse institutionnelle de Lourau, et d'hypothèses suggérées par Barel, et se heurte aux tentatives violentes de l'exécutif départemental d'empêcher ses investigations.

Le livre se termine par des enquêtes sur les routes et autoroutes françaises de Narbonne à Avignon auprès des femmes, accompagnées ou non de parentèles, qui poursuivent leur transmigration européenne en rejoignant les nations nordiques permissives ou en retournant chez elles. Ses franchissements fréquents des frontières, entre les emplacements de marchandisation des femmes et ceux de leur vie avec leurs parentèles d'accompagnement, donnent lieu à des récits d'une anthropologie des allers-retours vers une humanité ordinaire de ces femmesmarchandises.

AlainTarrius nous livre un récit passionnant et inquiétant. Des chercheurs, des travailleurs sociaux, des étudiants et toute autre personne curieuse des continuités entre mondes souterrains et officiels trouveront là une forte inspiration, à l'identique de travaux deTrascher ou de Park souvent cités par le chercheur.

Il est peut-être regrettable que ne soient pas davantage utilisées les formalisations permises par l'analyse des réseaux : le constat d'insécabilité des rapports économiques et affectifs, convaincant pour comprendre les rapports sociaux dans les territoires circulatoires et en exposer une anthropologie est bien moins évident dans ces univers de logiques marchandes où les femmes elles-mêmes sont marchandises.

Le lecteur de ce livre sera déçu par l'absence d'une recherche sur le retour des femmes au pays. Qu'en est-il de l'affirmation de l'auteur, au détour d'une phrase, selon laquelle " elles réalisent dans leur transmigration de six années l'ascension sociale que ne leur aurait jamais permis la sédentarité " ? Alain Tarrius compte bien apporter une réponse à cette question dans une prochaine recherche.

Fatima Oacha Maître de conférences en sociologie Université de Toulouse 2 Jean Jaurès LISST-CERS 
Coulibaly, Adama (dir.)

Konan, Yao Louis (dir.)

Les Écritures migrantes. De l'exil à la

migrance littéraire dans le roman francophone. -, Paris : L'Harmattan, 2015. - $250 \mathrm{p}$. ISBN : 978-2343-055-67-1

Le concept d'écriture migrante, apparu au Québec dans les années 1980, s'applique à de nombreux écrivains émigrés tels Alain Mabanckou, Calixte Beyala, Patrice Naganang et ceux qui sont analysés dans l'ouvrage collectif ici recensé, Tierno Monénembo, Marie Cardinal, Alain Vircondelet, Fatou Diome, Abdourahman Waberi, Nancy Huston, Gisèle Pineau, Leila Sebbar, auteurs africains, antillais, piedsnoirs.

L'écriture migrante est fondée sur un triptyque, le trauma du départ, la mobilité et enfin l'intégration dans un pays d'accueil, mais cette écriture se décline en une multitude de nuances. Dans les œuvres concernées, le pays d'origine est convoqué sous la plume de l'écrivain ; les paysages, la langue, les traditions constituent autant de souvenirs généralement bons et émouvants, parfois négatifs. L'évocation nostalgique de la terre natale peut remplir diverses fonctions : doloriste, car elle entretient les blessures du départ, cathartique, quand elle évacue les angoisses et les mélancolies, thérapeutique, puisqu'elle réconforte face aux problèmes présents. L'écriture migrante se trouve, par définition, en prise avec la mobilité du sujet, mouvement physique, mais aussi culturel et identitaire. Les métaphores du déplacement sont transparentes dans les titres des œuvres d'Abdourahman Waberi, Rift routes rails (2001) et Transit (2003), dans l'évocation fréquente des moyens de transport et des lieux de transit comme les aéroports. Les personnages des romans rêvent d'espaces réels ou fantasmatiques, ils se trouvent toujours entre deux frontières, juridiques ou psychiques ; parfois l'espace devient lui-même une sorte ce personnage ou d'incarnation du destin.
La métamorphose identitaire constitue un des thèmes fondamentaux du roman migrant. Le personnage arrive dans un nouveau pays, généralement la France, avec ses valeurs, sa langue, ses habitudes qui doivent brutalement cohabiter avec une autre culture, celle-là largement majoritaire. Les conséquences de cette confrontation se révèlent variables, en fonction du passé vécu, de l'expérience nouvelle allant de l'indifférence au mépris et au racisme. Les personnages réagissent diversement: repli sur eux-mêmes, confusion, effacement des points de repère, déchirure existentielle pouvant conduire à la folie, en tout cas partage identitaire. Dans Le Ventre de I'Atlantique (2003), Fatou Diome s'interroge : « Être hybride, I'Afrique et l'Europe se demandent, perplexes, quel bout de moi leur appartient. Je suis l'enfant présenté au sabre du roi Salomon pour le juste partage ". Certains reviennent au pays natal pour chercher une réponse et peut-être la paix. Espérance souvent illusoire, car ils ont évolué et restent d'éternels étrangers. D'autres trouvent un équilibre en se proclamant citoyens du monde ou en parvenant à unir les référents culturels de leurs deux terres. Parfois le salut vient de l'écriture ; celle-ci, dit Leila Sebbar dans Les Lettres parisiennes : histoires d'exil (1999), lui " tient lieu d'église ". Le cas le plus fréquent est le métissage identitaire ; ainsi Paris devient, sous la plume des critiques, "Afrique-sur-Seine ". Les écrivains introduisent de nombreux mots étrangers dans leur prose française, créoles, anglais, peuls, selon l'origine de l'auteur. En définitive, ballotés entre I'ailleurs et l'ici, les personnages vivent un état que les critiques nomment la " migritude ", état qui efface les frontières et, d'une certaine manière, tend vers l'universalité. "Paris n'est pas le centre du monde, mais bien le centre de la traversée de nos illusions ", observe Adama Coulibaly.

Le livre se montre très disert sur tous ces aspects. L'introduction, un peu jargonnante, risque de rebuter les lecteurs non spécialistes de critique littéraire. En 
revanche, les divers chapitres illustrent bien l'entre-deux identitaire que nourrit la migration. Les auteurs, plus ou moins autobiographes, en montrent la traduction dans le psychisme et les comportements. Ils apportent ainsi leur contribution à l'étude d'un phénomène complexe et multiforme.

Ralph Schor

Professeur émérite d'histoire Université de Nice-Sophia-Antipolis

Opeskin, Brian (éd.)

Perruchoud, Richard (éd.)

Redpath-Cross, Jillyanne (éd.)

Le droit international de la migration. -, Genève : Éditions Yvon Blais et Schulthess, 2014. $-575 \mathrm{p}$.

ISBN : 978-3725-569-54-0

Loin d'un intérêt cantonné à un champ disciplinaire, les règles juridiques applicables aux migrations méritent d'être prises en compte par un grand nombre de travaux de recherche relatifs aux phénomènes migratoires. En effet, ces ensembles normatifs participent à un double jeu d'influences, au niveau des acteurs publics, mais aussi à celui des acteurs privés. Élaborés par les États et par les organisations internationales, ils sont tout autant des produits que des encadrements des politiques migratoires. Plus ou moins intégrés par les migrants, ils peuvent influencer leurs comportements, tantôt dans une volonté de contournement des normes, ou au contraire de recherche de références protectrices.

Cependant, alors qu'un grand nombre des questions liées aux migrations implique simultanément, ou successivement, différents États, non seulement les normes internationales restent relativement peu nombreuses, mais en plus très peu de publications leur sont consacrées. Aussi la rencontre, à Genève en juin 2011, de dix-sept spécialistes des migrations pour un atelier consacré au droit international de la migration, était-elle particulièrement bienvenue. Cette manifestation a notamment abouti à la publication d'un ouvrage intitulé Foundations of International Migration Law (2012, Cambridge University Press, 494 p.), dont Le droit international de la migration est la traduction. En tant que francophone on ne pourra que se réjouir de cette initiative, rendue en grande partie possible grâce à l'aide financière de l'Organisation internationale pour les migrations (OIM). Par ailleurs, la contribution de cette institution internationale ne se limite pas à ce soutien matériel puisque l'on compte plusieurs de ses fonctionnaires parmi les auteurs.

Malgré les deux années qui séparent leur publication, il s'agit bien de deux versions linguistiques d'un unique recueil de contributions. Aussi pourra-t-on peut-être regretter que ce délai n'ait pas pu être mis à profit pour une actualisation. Ce d'autant plus que pour certains thèmes, comme les migrations des mineurs non accompagnés par exemple, un tournant important a été marqué au cours de cette période par l'adoption, le 5 avril 2011, de l'arrêt Rahimi contre Grèce, par la Cour européenne des droits de I'homme. Un commentaire de Jacqueline Bahbah, auteur de la contribution "Femmes, enfants et autres groupes de migrants marginalisés ", aurait été particulièrement intéressant.

La langue française n'empêchera pas les lecteurs de percevoir immédiatement l'influence nettement anglo-saxonne de la très grande majorité des travaux rassemblés - exceptions faites de Vincent Chetail et Richard Perrichoud - que ce soit dans la conception même du thème ou dans les références bibliographiques qui restent exclusivement anglophones.

Économistes, juristes, essentiellement universitaires, mais également praticiens offrent un panel riche et diversifié des aspects juridiques de ces thèmes. L'ensemble des questions migra- 
toires susceptibles d'être saisies par le droit international est abordé, offrant là un panorama certainement unique en son genre, au moins dans la littérature francophone. Alternant approches très générales ( "Tendances contemporaines de la migration internationale $»)$, souvent théoriques (" Étude théorique du droit international de la migration "), parfois très pédagogiques (notamment " Sources du droit international de la migration »), et études plus pointues (" Droit international du commerce et mobilité de maind'œuvre "), cet ouvrage semble mettre en évidence l'existence d'une branche spécifique du droit international appliqué à la migration. Un parallèle pourrait ainsi aisément être établi avec une intéressante initiative de la Faculté de droit de la Georgetown University qui a abouti à une Déclaration des droits des migrants au printemps 2010 (International Migrants Bill of Rights). Lors des différentes discussions sur la préparation de ce texte - élaboré par des étudiants et des universitaires de la Georgetown University, mais aussi de I'Université américaine du Caire et de I'Université hébraïque de Jérusalem -, la question de la définition du concept de migrants avait été fréquemment soulevée. Et, loin de n'être qu'une formalité terminologique, cette interrogation met en évidence la spécificité, ou les limites, de l'approche commune à cette Déclaration des droits des migrants et à l'ouvrage Le droit international de la migration. Peut-on considérer qu'être migrant réunit des individus par-delà les différences de leurs statuts juridiques, de leurs nationalités, de leurs situations géographiques, de leurs conditions de vie?

Certes, cet ouvrage ne prétend pas que le droit international de la migration recouvre une réalité uniforme, puisque I'avant-propos en particulier insiste sur l'absence de traité global et évoque un cadre juridique morcelé. Cependant, le choix de décliner dans le titre " droit international " et " migration " au singulier est caractéristique d'une approche dominante dans la doctrine anglo-saxonne qui peut certainement séduire, sans pour autant pleinement convaincre.

Au-delà de cette éventuelle réticence, on doit saluer cette entreprise particulièrement originale et l'opportunité qu'elle offre de découvrir des thématiques trop peu connues tant des spécialistes des migrations que des juristes. En effet, ces contributions sont accessibles aux non-spécialistes, ce qui n'est pas le moindre de leur mérite. Elles permettent de dépasser l'assimilation souvent faite entre droit(s) des migrations et droits des étrangers, témoignant alors de la pluralité des approches juridiques des phénomènes migratoires. À ce titre, on s'arrêtera en particulier sur l'étude de l'annexe de l'accord général sur les services de l'Organisation mondiale du commerce (OMC) consacrée aux mouvements des personnes physiques fournissant des services. II s'agit d'hypothèses (encore) peu développées, notamment si l'on compare le nombre d'individus potentiellement concernés avec celui des réfugiés. Néanmoins, elle vient souligner que la protection des droits de l'homme n'est pas l'unique branche du droit international à prendre en compte les migrations. D'autres contributions témoignent des liens entre la protection des droits de I'homme et des préoccupations économiques. Tel est notamment le cas des propos relatifs à la migration internationale de main-d'œuvre qui rappelle au passage l'ancienneté de l'action de l'Organisation internationale du travail (OIT) en la matière. Enfin, l'étude des tendances contemporaines souligne l'extrême rareté des travaux consacrés à la migration des parents âgés qui demeurent également les grands absents des réglementations du regroupement familial.

Marie-Françoise Valette Maître de conférences en droit public Université de Poitiers/MIGRINTER 